\title{
Application Effect Evaluation of Telmisartan combined with Spironolactone after Catheter Ablation of Patients with Paroxysmal Atrial Fibrillation
}

\author{
Rongcheng Zhao*, Jia Han, Lei Zhao \\ Department of Cardiovascular Diseases, Cangzhou Central Hospital, Cangzhou 061001, China
}

Funding: Observational study on effect of transcatheter radiofrequency ablation on patients with different types of atrial fibrillation (Project No.: 172302089)

\begin{abstract}
[Abstract] Objective: To evaluate the application effect of telmisartan combined with spironolactone after catheter ablation of patients with paroxysmal atrial fibrillation. Methods: 80 cases of patients with paroxysmal atrial fibrillation who received radiofrequency catheter ablation treatment from March 2013 to March 2016 in our hospital were randomly selected, these patients were divided into two groups according to the treatment methods, namely, the telmisartan with Spironolactone treatment group (combined treatment group, $\mathrm{n}=40)$ and the conventional therapy group $(\mathrm{n}=40)$. The hs-CRP, NT-proBNP, LAD and recurrence of the two groups were analyzed. Results: The hs-CRP, NT-proBNP levels after 3 months of the combined treatment group were significantly lower $(P<0.05)$, the recurrence rate $10.0 \%(4 / 40)$ was significantly lower than the conventional therapy group $27.5 \%(11 / 40)(P<0.05)$, the time to recurrence was significantly longer than the conventional therapy group $(P<0.05)$. Conclusion: The application effects of telmisartan combined with spironolactone after catheter ablation in the treatment of patients with paroxysmal atrial fibrillation are better than conventional therapy.
\end{abstract}

Key words: Paroxysmal atrial fibrillation; Catheter ablation; Telmisartan combined with spironolactone; Application effect

Publication date: May, 2021; Publication online: 31 May, 2021

*Corresponding author: Rongcheng Zhao, 896221780@.qq.com

\section{Introduction}

Auricular fibrillation (AF, referred to as atrial fibrillation) is a common arrhythmia. It is caused by the atrial-dominant reentrant loop induced atrial arrhythmia. The clinical manifestations include increased heart rate, chest tightness, palpitations, fatigue, and shortness of breath. Auricular fibrillation is a kind of arrhythmia which is extremely common in clinical practice, referred to as atrial fibrillation. It is divided into six types, namely, initial, paroxysmal, persistent, long-term persistent, permanent, and asymptomatic atrial fibrillation ${ }^{[1]}$. In China, atrial fibrillation has reached a total prevalence of about $0.77 \% \%^{[2]}$. Catheter radiofrequency ablation and drug therapy are the main modalities used in the clinical treatment of paroxysmal atrial fibrillation. At this stage, more and more patients opt for radiofrequency ablation surgery. In recent years, follow-ups revealed that some patients will have atrial fibrillation recurrence after surgery ${ }^{[3]}$. Therefore, the academic community are paying full attention to postoperative atrial fibrillation recurrence in order to take effective measures to promote a significant reduction in the recurrence rate of atrial fibrillation. Studies have shown that renin-angiotensin-aldosterone system (RAAS) blockers can reverse the structural and electrical remodelling of the atria, thereby reducing the incidence of new atrial fibrillation to the lowest extent ${ }^{[4]}$. This study statistically analyzed the clinical data of 80 patients with paroxysmal atrial fibrillation 
treated by catheter radiofrequency ablation in our hospital from March 2013 to March 2016 to evaluate the treatment effects of telmisartan combined with spironolactone on patients with paroxysmal atrial fibrillation after catheter radiofrequency ablation.

\section{Information and methods}

\subsection{General information}

From March 2013 to March 2016, 80 patients with paroxysmal atrial fibrillation treated by catheter radiofrequency ablation in our hospital were randomly selected.

\subsubsection{Inclusion criteria}

(1) Patients aged 45 to 70 years old;

(2) No restrictions on gender;

(3) Patients with paroxysmal atrial fibrillation;

(4) Grade I or II according to ASA Classification.

\subsubsection{Exclusion criteria}

(1) Patients with atrial fibrillation caused by other transient factors;

(2) Patients with valvular heart disease;

(3) Patients with bradyarrhythmia;

(4) Patients with abnormal thyroid functions;

(5) Patients with liver and kidney dysfunction;

(6) Patients with myocardial infarction in the past 3-6 months;

(7) Patients with contraindications to the use of Spirin;

(8) Patients who cannot take care of themselves;

(9) Patients with malignant tumors.

\subsubsection{Reasons for loss of contact and countermeasures}

\subsubsection{Expected reasons}

(1) The patient dropped out of the trial;

(2) The patient was transferred to other hospitals during the trial.

\subsubsection{Countermeasures}

Test patients were added to the test groups according to the ratio of $1: 1$.

\subsubsection{Ethics considerations}

(1) The patients and their immediate family members signed the informed consent form to participate in the clinical study;

(2) Fully guarantee the medical treatment and safety of the patients;

(3) Protect the privacy of patients;

(4) The trial protocol was registered in the Chinese Clinical Trial Registry, and the trials followed the "Ottawa Group
Statement for Clinical Trial Registration".

\subsubsection{Strictly follow double-blind principle.}

(1) Researchers were divided into 4 groups: the first group was responsible for screening and random allocation of test subjects; the second group was responsible for the treatment; the third group was responsible for data collection of observation indicators data; the fourth group was responsible for data sorting, statistical analysis and paper writing.

(2) The grouping of the test subjects is strictly confidential: neither the test subjects nor the researchers who perform the specific treatments were aware of the specific groupings of the test subjects.

(3) The researchers of the 4 research groups kept their operations confidential from each other.

\subsection{Grouping of test subjects}

According to the treatment method, these patients were divided into two groups, namely telmisartan/spironolactone combined treatment group (combined treatment group, $\mathrm{n}=40$ ) and conventional treatment group $(n=40)$.

\subsection{Treatment methods}

The patient's electrolytes, thyroid function, and transesophageal echocardiography were checked before the surgery, and the patient's blood was drawn 1 day before the surgery for testing of high-sensitivity $\mathrm{C}$-reactive protein (hs-CRP) and N-terminal Pro-B Type Natriuretic Peptide (NT-proBNP). Ablation of the peripheral pulmonary vein was given to the patients to achieve electrical isolation. If the patient had focal atrial arrhythmia, etc. during the surgery, additional ablation at the relevant sites would be administered. Then, patients in the conventional treatment group were given conventional treatment, and the patients were given amiodarone and warfarin according to the conventional treatment scheme to effectively prevent and avoid drugs that may affect the inflammatory response, such as statins and non-steroidal anti-inflammatory drugs; patients in the combined treatment group were treated with telmisartan combined with spironolactone on the basis of the above treatment scheme. The initial dose of telmisartan was $20 \mathrm{mg}$ per day. If the patient has hypertension, the target dose was set at $160 \mathrm{mg}$ per day, and if the patient has normal blood pressure, the target dose was set at $80 \mathrm{mg}$ per day. The dose of spironolactone was $20 \mathrm{mg}$ per day.

\subsection{Observation indicators}

1 and 3 months after the operation, the two groups of patients were urged to return to the hospital for follow-up visits, and the hs-CRP, NT-proBNP and 24h Holter ECG 
examinations were carried out, and their blood pressure, heart rhythm, and left atrial inner diameter (LAD) were recorded in detail and the medication was adjusted in time. The two groups of patients were regularly followed up through phone calls. If the patient has palpitations, he/she would be examined by routine ECG as soon as possible to confirm the recurrence of atrial fibrillation, that is, if the patient has atrial flutter/ atrial fibrillation/ atrial tachycardia for 30 s and above at 3 months after the surgery, indicating its early recurrence ${ }^{[5]}$.

\subsection{Statistical analysis}

The statistical software package SPSS20.0 was used in the process of statistical analysis of the data. Measurement data is expressed as ( $X \pm_{s}$ ), recurrence time was compared using Kaplan-Meier survival analysis, independent sample t-test was applied to inter-group comparisons, $\chi 2$ test was applied to count data, and significance level $\alpha=0.05$.

\section{Results}

\subsection{Comparison of the general information of the two groups of patients}

The general information of the subjects in each group were compared, and the results showed no statistical difference $(\mathrm{P}>0.05)$. In addition, during the course of the study, all subjects completed all trials. There were no dropouts or switch to other therapies (Table 1).

Table 1. Comparison of the general information of the two groups of patients

\begin{tabular}{ccccccc}
\hline \multirow{2}{*}{ Group } & \multirow{2}{*}{ No. Of Cases } & \multicolumn{2}{c}{ Gender Ratio } & \multirow{2}{*}{ Average Age (y/o) } & \multicolumn{2}{c}{$\begin{array}{c}\text { Average Disease Course } \\
\text { (yrs) }\end{array}$} \\
\cline { 3 - 5 } & & Male & Female & & \\
\hline Combination & 40 & $25(62.5)$ & $15(37.5)$ & $62.3 \pm 4.5$ & $2.6 \pm 0.8$ \\
Conventional & 40 & $26(65.0)$ & $14(35.0)$ & $63.4 \pm 4.3$ & $2.8 \pm 0.6$ \\
\hline
\end{tabular}

3.2 Comparison on the changes in hs-CRP, NT-proBNP, LAD levels of the two groups of patients at 1 and 3 months after surgery

Intra-group comparison, the hs-CRP level of patients in the combined treatment group at 3 months after surgery was significantly lower than at 1 month after surgery $(\mathrm{P}<0.05)$, and the level of NT-proBNP at 3 months after surgery was significantly lower than that of 1 month after surgery $(\mathrm{P}<0.05)$. However, the difference in LAD level at 1 and 3 months after surgery was not significant $(\mathrm{P}>0.05)$; the NT-proBNP level of patients in the conventional treatment group at 3 months after surgery was significantly lower than that at 1 month after surgery $(\mathrm{P}<0.05)$. However, the differences in hs-CRP and LAD levels at 1 and 3 months after operation were not significant $(\mathrm{P}>0.05)$. Inter-group comparison, there was no significant difference in the hs-CRP, NT-proBNP and LAD levels between the two groups at 1 month after surgery ( $\mathrm{P}>0.05)$, and the hs-CRP, NT-proBNP levels in the combined treatment group at 3 months after surgery were significantly lower than those of the conventional treatment group $(\mathrm{P}<0.05)$, but the difference in LAD between the two groups was not significant $(\mathrm{P}>0.05)$ (Table 2).

Table 2. Comparison on the changes in hs-CRP, NT-proBNP, LAD levels of the two groups of patients at 1 and 3 months after surgery $(x+s)$

\begin{tabular}{|c|c|c|c|c|c|}
\hline Group & No. of Cases & Time & hs-CRP（mg/L） & NT-proBNP（pg/ml） & LAD（ $\mathbf{m m})$ \\
\hline \multirow[t]{2}{*}{$\begin{array}{l}\text { Combined } \\
\text { Treatment }\end{array}$} & 40 & 1 month after surgery & $2.12 \pm 0.4$ & $128.78 \pm 41.32$ & $34.53 \pm 1.79$ \\
\hline & & 3 months after surgery & $1.56 \pm 0.7^{\#}$ & $75.9 \pm 28.11^{\# *}$ & $34.67 \pm 6.60$ \\
\hline \multicolumn{4}{|l|}{ Conventio } & $135.45 \pm 67.76$ & $34.28 \pm 1.62$ \\
\hline \multicolumn{6}{|l|}{ Treatment } \\
\hline & & 3 months after surgery & $2.01 \pm 0.3$ & $107.09 \pm 74.53^{\#}$ & $34.81 \pm 3.56$ \\
\hline
\end{tabular}

Note: compared with the same group itself at 1 month after surgery, ${ }^{\#} P<0.05$; compared with the conventional treatment group, ${ }^{*} P<0.05$. 


\subsection{Hemodynamic changes of the test subjects}

The subjects' MAP and HR Levels: Compared with pre-surgery, the levels of both groups increased at 1 month and 3 month after surgery. The combined treatment group was higher than the conventional treatment group at the same time-point after surgery $(\mathrm{P}<0.05)$ (Table 3, Table 4).

Table 3. The changes in the MAP level of the two groups of patients $\left(\bar{x}_{ \pm \mathrm{S}}, n=16\right)$

\begin{tabular}{|c|c|c|c|c|}
\hline Group & No. of Cases & Pre-surgery & 1 month after Surgery & 3 months after Surgery \\
\hline Combined Treatment & 40 & $93.65 \pm 6.88$ & $103.53 \pm 5.85^{\#^{*}}$ & $109.61 \pm 5.27^{\#^{*}}$ \\
\hline Conventional Treatment & 40 & $92.18 \pm 6.85$ & $96.02 \pm 11.36^{\#}$ & $102.25 \pm 7.58^{\#}$ \\
\hline
\end{tabular}

Note: compared with the same group itself at 1 month after surgery, ${ }^{\sharp} P<0.05$; compared with the conventional treatment group, ${ }^{*} P<0.05$.

Table 4. The changes in the HR level of the two groups of patients ( $\left.\overline{\boldsymbol{x}}_{ \pm \mathrm{S}}, n=16\right)$

\begin{tabular}{|c|c|c|c|c|}
\hline Group & No. of Cases & Pre-surgery & $\mathrm{T} 2$ & $\mathrm{~T} 4$ \\
\hline A & 40 & $75.36 \pm 12.26$ & $88.23 \pm 11.52^{\#^{*}}$ & $95.22 \pm 12.36^{\#^{*}}$ \\
\hline $\mathrm{C}$ & 40 & $74.18 \pm 13.05$ & $78.15 \pm 12.58^{\#}$ & $84.52 \pm 14.82^{\#}$ \\
\hline
\end{tabular}

Note: compared with the same group itself at 1 month after surgery, ${ }^{\sharp} P<0.05$; compared with the conventional treatment group, ${ }^{*} P<0.05$.

3.4 Comparison on the recurrence status of the two groups of patients at 3 months after surgery

The recurrence rate of patients in the combined treatment group at 3 months after surgery was $10.0 \%$ (4/40), which was significantly lower than that of the conventional treatment group, $27.5 \%(11 / 40)(\mathrm{P}<0.05)$, and the recurrence time was significantly longer than that of the conventional treatment group $(\mathrm{P}<0.05)$ (Table 5).

Table 5. Comparison on the recurrence status of the two groups of patients 3 months after surgery

\begin{tabular}{cccc}
\hline Group & No. of Cases & Recurrence & Recurrence Time (d) \\
\hline Combined Treatment & 40 & $4(10.0)$ & $85.3 \pm 10.2$ \\
Conventional Treatment & 40 & $11(27.5)$ & $77.8 \pm 10.0$ \\
\hline
\end{tabular}

Note: compared with conventional treatment group, ${ }^{*} \mathrm{P}<0.05$.

\section{Discussion}

Currently, the drug treatment after radiofrequency catheter ablation of atrial fibrillation mainly includes conventional antiarrhythmic drugs, statins and RAAS blockers. Upstream treatments such as RAAS blockers directly act on the atrial fibrillation matrix, participate in the improvement of electrical and structural remodeling, and reduce the risk of recurrence after atrial fibrillation, which is a hot research topic. RAAS is the main drug for clinical treatment of patients with atrial fibrillation after catheter radiofrequency ablation and it plays an extremely important role in atrial remodeling. A large number of relevant medical scholarshave shown that in the process of primary and secondary prevention of atrial fibrillation, angiotensin-converting enzyme inhibitors and angiotensin receptor blockers play an extremely important role, especially angiotensin-converting enzyme inhibitors, which has a more significant effect in promoting the reduction of the recurrence rate of atrial fibrillation ${ }^{[6-9]}$. Meanwhile, aldosterone plays an important role in RAAS, and the occurrence of atrial fibrillation is also directly and profoundly affected by it. Patients with atrial fibrillation have a higher serum aldosterone concentration, and spironolactone is an aldosterone inhibitor, which can effectively prevent atrial fibrosis, thereby reducing the incidence of atrial fibrillation to the minimal extent ${ }^{[10]}$.

Studies have shown that hs-CRP, NT-proBNP, and LAD all play an extremely important role in the prediction of atrial fibrillation recurrence ${ }^{[11]}$. LAD can be used as an important indicator in predicting the risk of recurrence of atrial fibrillation after ablation, because it can reflect the remodeling of atrial structure to a certain extent. Atrial fibrillation patients with high NT-proBNP levels before 
catheter radiofrequency ablation accounted for nearly $70 \%$ of the total, and were linearly correlated with LAD level. NT-proBNP can be used as an extremely important independent factor in the prediction of atrial fibrillation recurrence. This is because patients without recurrence of atrial fibrillation have significantly lower NT-proBNP levels after surgery compared with pre-surgery. hs-CRP is a very typical inflammatory indicator, which can be used as an extremely important indicator in the prediction of the long-term recurrence of atrial fibrillation. This is because it may be involved in clearing the death cells during the remodeling of the central atrial structure in the process of atrial fibrillation, thereby affecting the left atrium matrix, and at the same time promote a large increase in the incidence of non-venous atrial fibrillation. Telmisartan is a new type of angiotensin-converting enzyme, which can fully inhibit RAAS when combined with spironolactone, providing a good prerequisite for the reduction of calcium overload in myocardial cells, thereby promoting the reduction of atrial electrical remodeling, and decrease in left atrial and left ventricular pressure and plasma NT-proBNP level ${ }^{[12]}$. The results of this study showed that the NT-proBNP level of the combined treatment group was significantly lower than that of the conventional treatment group 3 months after the surgery, the recurrence rate was significantly lower than that of the conventional treatment group, and the recurrence time was significantly longer than that of the conventional treatment group, which fully confirmed this point.

In conclusion, the efficacy of telmisartan/ spironolactone combined treatment after catheter radiofrequency ablation in patients with paroxysmal atrial fibrillation is better than conventional treatment, and it is more effective in reducing the levels of hs-CRP and NT-proBNP and early recurrence rate in patients 3 months after surgery, and prolonging the recurrence time of patients, which is worthy of promotion and application in clinical treatment.

\section{References}

[1] Li XM. Changes of vitality structure of left atrium and left ventricle after radiofrequency ablation in patients with chronic atrial fibrillation[J]. Journal of Clinical and Experimental Medicine, 2014, 13(4): 285-287.
[2] Cao TF, Tao XL, Zhang XR, et al. Case-control study on serum lipid levels in patients with and without atrial fibrillation[J]. Journal of Third Military Medical University, 2013, 35(15): 1604-1605.

[3] Li KL, Zheng J, Zhang CY. The associations between early recurrence of paroxysmal atrial fibrillation after catheter ablation and C-reactive protein[J]. Journal of Clinical Cardiology, 2014, 30(3): 195-197.

[4] Wang HY. An analysis of misdiagnosis in the electrophysiologic study and radiofrequency catheter ablation[J]. China Foreign Medical Treatment, 2014, 8(5): 178-179.

[5] Hu LQ, Xue YM, Zhan XZ. Combined therapy with telmisartan and spironolactone prevents early recurrence after catheter ablation for paroxysmal atrial fibrillation[J]. International Journal of Cardiovascular Disease, 2014, 41(2): 124-126.

[6] Shen QM, Liu FY. Related factors for late recurrence after catheter ablation for 80 patients with paroxysmal atrial fibrillation $[\mathrm{J}]$. Chinese Journal of Clinical Healthcare, 2013, 16(3): 268-270.

[7] Cui YX, Yang SX. The advance of atrial fibrillation and atrial remodeling $[\mathrm{J}]$. Chinese Journal of Cardiovascular Review, 2014, 12(10): 944-949.

[8] Wang C, Gu TX, Xiu ZY, et al. The long-term outcome of surgical treatment for atrial fibrillation with modify maze procedure by radiofrequency ablation during valve surgery $[\mathrm{J}]$. Chinese Journal of Cardiovascular Review, 2013, 11(1): 9-12.

[9] Wang LF, Luo J, Ji QJ, et al. Effect of spironolactone on electrical remodeling induced by rapid atrial pacing in rabbits[J]. Chinese Journal of Gerontology, 2012, 32(6): 1205-1207.

[10] Huang CX, Zhang S, Ma CS, et al. Current knowledge and management recommendations of atrial fibrillation 2012[J]. Chinese Journal of Cardiac Arrhythmias, 2012, 16(4): 246-289.

[11] Xing AJ, Hou LJ, Meng LK, et al. Analysis of the prevalence and risk factors of atrial fibrillation in female population of Kailuan Group[J]. Hebei Medical Journal, 2015, 37(2): 276-278.

[12] Gao SC. Clinical observation on the antithrombotic effect of different antithrombotic schemes in patients with chronic atrial fibrillation[J]. Hebei Medical Journal, 2013, 35(18): 2822-2823. 